\title{
Towards polarimetry as a tool for the detection of extra-terrestrial life
}

\author{
Stefano Bagnulo ${ }^{1}$, Michael F. Sterzik ${ }^{2}$ and Alberto Cellino ${ }^{4}$ \\ ${ }^{1}$ Armagh Observatory, College Hill, Armagh BT1 9DG, UK. e-mail: sba@arm.ac.uk \\ ${ }^{2}$ European Southern Observatory, Karl-Schwarzschildstrasse 2, D-85748, Garching bei \\ München, Germany. e-mail: msterzik@eso.org \\ ${ }^{3}$ INAF - Osservatorio Astrofisico di Torino, Pino Torinese. e-mail: cellino@oato.inaf.it
}

\begin{abstract}
Linear broadband polarimetry is used to characterize the objects of our solar system, and has also been proposed as a diagnostic tool for the atmospheres of exo-solar planets. Homochirality characterizes life as we know it and induces circular polarization in the diffuse reflectance spectra of biotic material. Hence it has been suggested that circular polarimetry may be used as a remote sensing tool for the search of extra-terrestrial life. With this motivation in mind we have decided to explore the potential of both linear and circular spectropolarimetry as a diagnostic tool for remote sensing of biotic material. We have used the calibration unit of the EFOSC2 instrument of the La Silla Observatory to obtain low resolution, but high signal to noise circular and linear spectropolarimetric measurements of a number of inorganic and organic materials. We then compare our "laboratory data" with spectropolarimetric observations of atmosphere-less bodies of our solar system and of Earthshine obtained with instruments very similar to that one used for our laboratory samples. We conclude that linear polarization measurements are more suitable than circular polarization measurements for the characterization of planetary surfaces and atmospheres, and for the search of extra-terrestrial life.
\end{abstract}

Keywords. astrobiology, polarization, minor planets, asteroids

\section{Introduction}

Linear polarimetry may be used for the characterization of the atmospheres of exoplanets. Since light reflected by a planet is polarized, while the radiation coming from the host star is not, polarimetry may work as a contrast technique, potentially capable of masking the stellar radiation, hence disentangling the signal coming from the planet from the overwhelming radiation of the host star.

Perhaps the most important next goal of Astronomy is the detection of extra-terrestrial life. Can polarimetry be used to characterize not only the atmospheres but also the surfaces of the exo-planets, and eventually be adopted as a detection tool for extraterrestrial life?

\section{Homochirality and circular polarization}

Homochirality, i.e., the exclusive use of L-amino acids and D-sugar in biological material, induces circular polarization in the diffuse reflectance spectra of biotic material. The reasons for homochirality in living material are unknown, but if it characterizes life elsewhere in the Universe, then biosignatures could be remotely sensed by circular polarimetry. Circular spectropolarimetric laboratory measurements of leaves and bacteria have been published by Sparks et al. (2009a,b) and by Sterzik et al. (2010), who identified signatures of chlorophyll pigments in both samples of leaves and cyanobacteria. An astronomical experiment was performed by Sparks et al. (2005), who obtained imaging 
circular polarimetry of the Martian surface during the favorable opposition of 2003 to seek evidence of anomalous optical activity. Their experiment led to a null result, and later, Sparks et al. (2012) suggested that circular spectropolarimetry of selected areas of the Mars surface could reveal chiral signatures. In this work we will discuss further options for detection of extraterrestrial life through polarimetry.

\section{The laboratory}

Key to the interpretation of polarimetric data may come from a comparison with laboratory data. For instance, Martin et al. (2008) have set up a dedicated laboratory to measure the elements of the Mueller matrices for a number of different organic and inorganic materials. We decided to make an experiment more closely linked to astronomical observations using the Faint Object Spectrograph and Camera (EFOSC2) of the ESO New Technology Telescope (NTT) at the La Silla Observatory (Chile). EFOSC2 is a focal reducer instrument capable of doing low resolution spectropolarimetry. We had the opportunity to perform these experiments in 2010, at a time when EFOSC2 was detached from the telescope during a run with a visitor instrument.

\subsection{Laboratory samples}

The samples we have decided to study include organic and inorganic material. Among organic material we considered a sample of cyanobacteria. Cyanobacteria are small bacteria and usually unicellular, but they often grow in colonies large enough to be seen. They are the oldest known fossils (more than 3.5 billion years old) and one of the largest and most important groups of bacteria on Earth. Their typical habitats are subsurface layers of halites (rock salt) and quartz rocks, which allow them to survive extreme desiccation and high doses of both ionizing and ultraviolet radiation, and make them an appropriate fiducial point for astrobiology. We have collected samples of Chroococcidiopsis in the Llamara salt-desert in Chile, and measured their linear and circular polarization. We have repeated similar measurements with philodendron leaves and inorganic material such as finely grinded quartz, and finally with quartz samples partially covered by colonies of bacteria.

\subsection{Laboratory data}

Figure 1 shows the results of our observations of a Philodendron leaf (left panel), and of a sample of Chroococcidiopsis fully covering the screen of the EFOSC2 internal calibration unit (mid panel) and partially filling some quartz stones (right panel). We must point out that our observations are not performed under laboratory controlled conditions, in particular we are not even able to define a value of the scattering angle, as our samples are illuminated through an integrating sphere flat-fielding unit. The conditions are probably not too far from back-scattering conditions, and the observed linear polarization signal is probably much lower than what would be observed in quadrature (i.e., when the angle between the illuminating source, the target and the observer is $90^{\circ}$ ). Also, since our observations are obtained by illuminating the samples with unpolarised radiation, we can measure only the diagonal elements of the Mueller matrices. Finally, the volume of bacteria in our samples, and the filling factors, are not defined. In conclusion, we must strongly point out that our results are only qualitative. Nevertheless, our experiments are of great interest as we are able to clearly see the differences in the polarization produced by the reflection from different kinds of surface. In particular, we have detected many narrow features due to the chlorophyll pigments in the samples of cyanobacteria and 

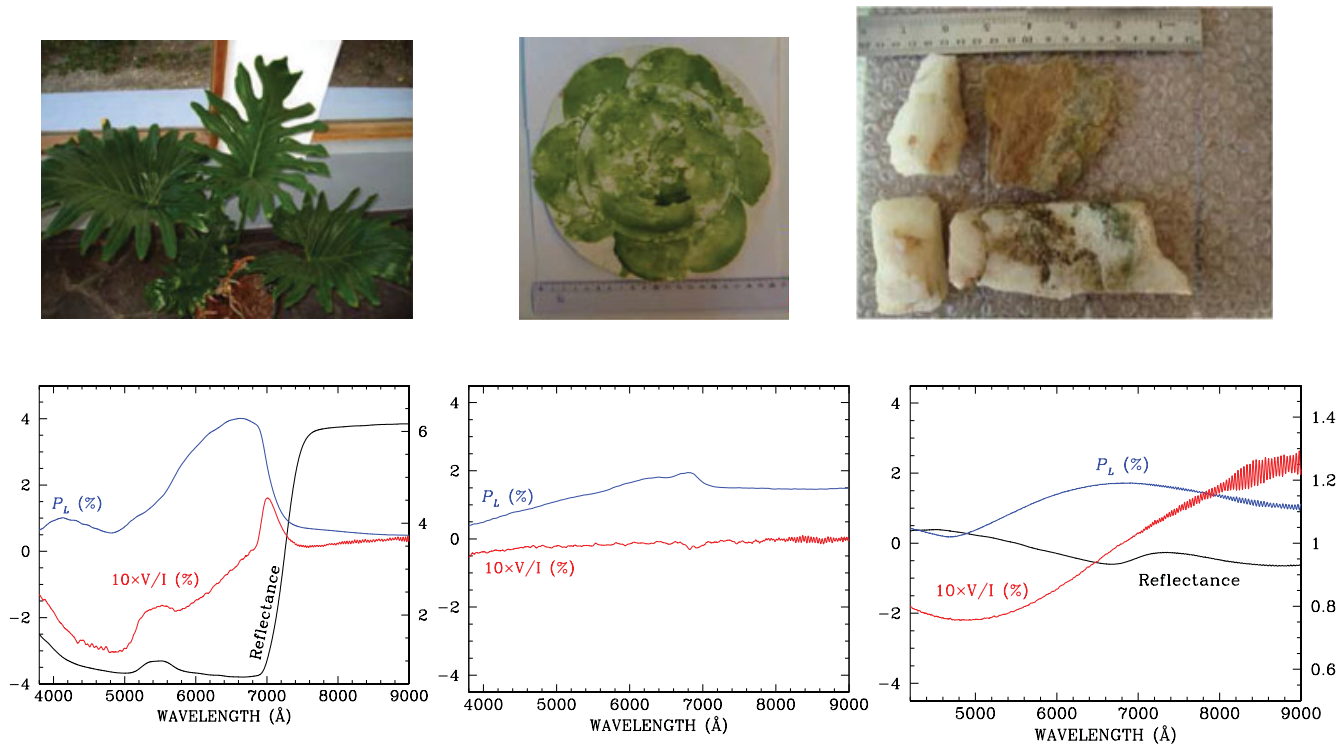

Figure 1. Reflectance, linear and circular polarization spectra of a sample of a philodendron leaf (left panel), a sample of Chroococcidiopsis (mid panel) and a sample of quartz partially covered by cyanobacteria (right panel). Scale for spectropolarimetric data is given in the left $y$-axis, while reflectance scale is on the right $y$-axis. Reflectance spectra are normalized to 1 at $\lambda=5500 \AA$. Red parts of the polarization spectra are strongly affected by fringing.

leaves, while nothing is detected in the quartz samples. Results of some of our circular polarimetric observations had been already published by Sterzik \& Bagnulo (2011).

\section{The astronomical instrumentation}

EFOSC2 is normally installed at the Nasmyth focus of the ESO New Technology Telescope (NTT), which can no longer be used for accurate astronomical linear polarimetric measurements. Currently, only few spectropolarimeters with good capabilities in the continuum are available to the community. The FOcal Reducer and low dispersion Spectrograph (FORS) at the Very Large Telescope (VLT) and the Intermediate dispersion Spectrograph and Imaging System (ISIS) at the William Herschel Telescope (WHT) are among them. Clearly none of these facilities can reach the signal-to-noise ratio necessary to search for life signatures in exo-planets. However, many bodies of our Solar System are within reach of these instruments, and some proof-of-concept experiments may be performed.

\subsection{Solar system objects}

Can astronomical polarimetry be used to identify organic material in extra-terrestrial bodies? Could polarimetry reveal if a planet hosts life? An interesting experiment is to obtain polarimetric observations of the only planet in which we are sure to find life. To simulate the observations of Earth as an exo-planet, ideally one would like to install a polarimeter on the Moon (or even farther away, so that disk-integrated signals can be measured), and point it to our planet. Waiting for this to happen (see Karalidi et al. 2012 for a description of the LOUP project), we have used the trick to look at the Earth using the Moon as a giant mirror, and obtained spectropolarimetric measurements of Earthshine. Sterzik \& Bagnulo (2009, 2011) have reported that circular 

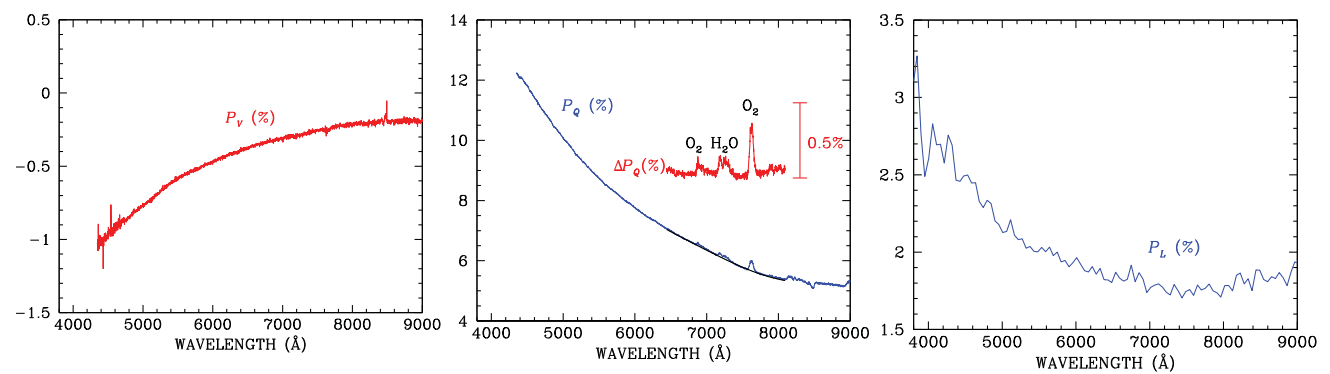

Figure 2. Circular and linear polarization spectra of Earthshine (left and mid panel, respectively), and linear polarization spectrum of S-type asteroid (433) Eros (right panel).

spectropolarimetry does not show any obvious hallmark of life. In fact, the observed signal is entirely dominated by a cross-talk effect from linear polarization, that has been extensively discussed by Bagnulo et al. (2009). The left panel of Fig. 2 shows circular polarization observations of Earthshine obtained during our 2011 campaign. More interesting results come from linear spectropolarimetry. Sterzik, Bagnulo \& Pallé (2012) have detected clear signatures of the atmosphere and surface of our planet, in particular the presence of oxygen and water, as well as the vegetation edge. The mid panel of Fig. 2 shows both the global behaviour of the polarization spectrum of Eartshine and the residuals obtained after subtracting the "continuum" polarization. The residuals clearly show the signature of $\mathrm{O}_{2}$ and $\mathrm{H}_{2} \mathrm{O}$ molecular lines. Sterzik, Bagnulo \& Emde (2015), report more recent results of a detailed modelling of Earthshine data. For comparison, the right panel of Fig. 2 shows the relatively featureless spectrum of S-type asteroid (433) Eros.

\section{Discussion and Conclusions}

In spite of the obvious interest offered by the theoretical possibility of detection of homochirality by means of circular polarization measurements, this approach clearly faces overwhelming problems of practical detectability of the expected features, which are probably too weak to measure in celestial bodies.

A more interesting approach is possibly offered by linear polarimetric measurements, as the observable features are substantially stronger, hence easier to detect. Nevertheless, our Earthshine observations suggest that atmospheric features such as those produced by water and oxygen molecules are predominant over more direct bio-signatures such as, e.g., chlorophyll-a pigment response around $680 \mathrm{~nm}$ and other pigments like carotinoids and phycocyanins.

\section{References}

Bagnulo, S., Landolfi, M., Landstreet, J. D., Landi Degl'Innocenti, E., Fossati, L., \& Sterzik, M. 2009, PASP 121, 993

Bagnulo, S., Cellino, A., \& Sterzik, M. F. 2015, MNRAS 446, L11

Karalidi, T., Stam, D. M., Snik, F., Bagnulo, S., Sparks, W. B., \& Keller, C. U. 2012, Planetary and Space Science 74, 202

Martin, W. E., Hesse, E., Hough, J. H., Sparks, W. B., Cockell, C. S., Ulanowski, Z., Germer, T. A., \& Kaye, P. H. 2008, JQSRT 111, 2444

Sparks, W. B., Hough, J. H., \& Bergeron, L. E. 2005, Astrobiology 5, 737

Sparks, W. B., Hough, J. H., Germenr, T. A. et al. 2009, PNAS 106, 7816

Sparks, W. B., Hough, J. H., Kolokolova, L., et al. 2009, JQSRT 110, 1771 
Sparks, W. B., Hough, J. H., Germer, T. A., Robb, F., \& Kolokolova, L. 2012, Planetary and Space Science 72, 111

Stam, D. 2008, A\&A 482, 989

Sterzik, M. F. \& Bagnulo, S., 2009, in: K. J. Meech, J. V. Keane, M. J. Mumma, J. L. Siefert, \& D. J. Werthimer (eds.), Bioastronomy 200\%: Molecules, Microbes and Extraterrestrial Life, ASP Conf. Series 449, 371

Sterzik, M., Bagnulo, S., Azua, A., Alfaro, J., \& Vicuna, R. 2010, The Messenger 142, 25

Sterzik, M. \& Bagnulo, S. 2011, in: P. Bastien, N. Manset, D. P. Clemens, \& N. St-Louis (eds.), Astronomical Polarimetry 2008: Science from Small to Large Telescopes, ASP Conf. Series 449, 399

Sterzik, M. F., Bagnulo, S., \& Pallé, E. 2012, Nature, 483, 64

Sterzik, M. F., Bagnulo, S., \& Emde, C. 2015, in: K. N. Nagendra, S. Bagnulo, R. Centeno, \& M. J. Martínez González (eds.) Polarimetry: From the Sun to Stars and Stellar Environments, Proc. IAU Symp. 305 (CUP), p. 305 